\title{
Parameters for a "Project-X"-based Muon Collider
}

\author{
David Neuffer
}

\author{
Fermilab \\ PO Box 500, Batavia IL 60510
}

\begin{abstract}
A set of parameters for a $4 \mathrm{TeV} \mu^{+}-\mu^{-}$Collider based on the use of "Project-X" as the proton source for $\pi$ production is presented. The scenario uses $56 \mathrm{GeV}$ protons from the "Main Injector", bunches these protons in a new buncher to $9 \sim 1 \mathrm{~m}$ long bunches. The estimated luminosity is $\sim 4 \times 10^{34} \mathrm{~cm}^{-2} \mathrm{~s}^{-1}$, with several potential upgrade possibilities.
\end{abstract}

\section{Introduction}

Fermilab is currently developing a proton plan for an upgrade of its proton source, which is currently called "Project-X".[1] This plan is based on the use of ILC rf cavities and cryomodules to form an $8 \mathrm{GeV} \mathrm{H}^{-}$linac. The $\mathrm{H}^{-}$linac is multiturn charge-exchange injected into the Recycler and the accumulated protons are then transferred into the Main Injector for acceleration up to $\sim 120 \mathrm{GeV}$, providing $>2 \mathrm{MW}$ of proton beam for neutrino production or other experiments. A key feature of the linac is that its parameters are fixed to correspond to the acceleration requirements of the ILC, which sets the current to 9ma with a $1 \mathrm{~ms}$ pulse length $\left(5.6 \times 10^{13}\right.$ particles $)$ at $5 \mathrm{~Hz}$. For Project $\mathrm{X}, 3 \mathrm{H}^{-}$linac pulses are accumulated in the Recycler and transferred to the Main Injector for acceleration. The main injector can cycle to $120 \mathrm{GeV}$ in $\sim 1.4 \mathrm{~s}$. At that intensity $\left(3 \times 1.7 \times 10^{14} \times 120 \mathrm{GeV} / 1.4 \mathrm{~s}\right)$ the beam power is 2.3 MW. In this mode 4 of the 7 linac cycles available every $1.4 \mathrm{~s}$ are available for other users; however, this is only $\sim 0.2 \mathrm{MW}$ of $8 \mathrm{GeV}$ beam power.

In the present paper we are considering whether Project $X$ could be adapted to feed the front end of a $\mu^{+}-\mu^{-}$Collider. In present discussions, the Collider needs $\sim 2$ MW of proton power, but that proton power must be bunched into a few relatively short bunches per second $(\sim 20$ $\mathrm{Hz}, \sim 1 \mathrm{~m}$ long bunches) and the optimum proton energy is somewhat less than $120 \mathrm{GeV}$. Some reconfiguration and/or additions to the baseline Project $X$ source are needed. Several such reconfigurations are possible. In the present note we develop a relatively attractive scenario that maximizes the use of the present Fermilab facilities. 


\section{GeV Proton Driver Scenario Overview}

In this scenario we maintain the linac intensity but use every linac pulse in the Collider pdriver. Thus after accumulating 3 linac pulses in the Recycler we would transfer them immediately to the Main Injector and accelerate and reset while the next three linac pulses are stacked in the Recycler (0.6s). Assuming the same acceleration rate as that used in the 120 $\mathrm{GeV}$ beam example, we find that the proportionate final proton energy is $56 \mathrm{GeV}$. The beam would then be single turn extracted into a Buncher ring of the same circumference. The Main Injector would be returned to $8 \mathrm{GeV}$ to receive the next Recycler (three-linac) pulse. The Buncher would form the $\sim$ full circumference Main injector bunch train into $\sim 9$ short bunches (in $\sim 0.2 \mathrm{~s}$ ) that would be extracted ( $\sim 1$ at a time) onto the $\pi$ production target to initiate the muon collider cycle. The $9 \pi^{+-}$bunches would form $9 \mu^{+}$and $9 \mu^{-}$bunches for the collider sequence. Since each $0.6 \mathrm{~s}$ cycle forms 9 bunches the collider operates at a fundamental $15 \mathrm{~Hz}$ rate. Parameters of that nominal cycle are presented in Table 1.

The number of muons per original proton is based on the cooling scheme presented in ref. [2], where the surviving cooled muons per initial $24 \mathrm{GeV}$ p was $\sim 0.4 \times 0.07=0.028$. The number per $56 \mathrm{Gev}$ proton is estimated to be larger by a factor of $56 / 24=2.333$, so $\mu / \mathrm{p}=0.065$. (Both $\mu^{+}$ and $\mu^{-}$are produced at that rate in the scheme.) The transverse emittance is the same that is obtained in the scenario $\left(\varepsilon_{\mathrm{t}}=25 \pi \mathrm{mm}-\mathrm{mrad}\right)$.

If the $\beta^{*}$ at collision is $0.003 \mathrm{~m}$, the beam sizes at collision would be $2 \mu$ in radius. The lifetime of 900 turns corresponds to a mean bending field of 6T. With these parameters the luminosity is $\sim 4 \times 10^{34} \mathrm{~cm}^{-2} \mathrm{~s}^{-1}$.

\section{Comments on Scenario}

The major addition to the Project $\mathrm{X}$ scenario is the $56 \mathrm{GeV}$ Buncher. In this scenario the buncher compresses the beam from the Main injector into 9 compressed bunches within < $\sim 0.2 \mathrm{~s}$. This would compress an $\sim 3320 \mathrm{~m}$ long beam into $9 \sim 4 \mathrm{~m}$ bunches. This is a compression factor of almost $\sim 100$, and would increase an energy spread of $\pm 2 \mathrm{MeV}$ to $\pm 200 \mathrm{MeV}$. The $\pm 2 \mathrm{MeV}$ is consistent with the expected energy spread of the linac, and would set an (approximate) lower limit on the longitudinal emittance. The large compression factor would be very difficult in practice.

We have simulated an rf scenario with the desired parameters, but the conditions are very idealized. We use a buncher ring the circumference of the Main Injector and $\gamma_{t}=21.8$ and a harmonic $9 \mathrm{rf}$ with linear bunching (sawtooth $\mathrm{rf}$ ). The $\mathrm{rf}$ is increased from 0 to $0.56 \mathrm{MV} /$ turn over a period of $0.167 \mathrm{~s}$, and at the end of this the rms bunch length is $1.35 \mathrm{~m}$ and the rms energy width is increased from $2 \mathrm{MeV}$ rms to $150 \mathrm{MeV}$ (full width $\pm 300 \mathrm{MeV}$ ). (Note: $\mathrm{h}=9$ is $0.81 \mathrm{MHz}$, and it is possible that $0.56 \mathrm{MV}$ of linear $\mathrm{rf}$ at this frequency is impractical.)

A somewhat better rf scenario is obtained if the beam is bunched in a smaller circumference ring. As an example we consider a buncher ring with $1 / 4$ the circumference of the Main Injector, with the buncher ring filled by transverse phase-space stacking. At parameters of $\gamma_{t}=$ 18 , a sawtooth $\mathrm{h}=9 \mathrm{rf}$ system of 0 to $0.4 \mathrm{MV} /$ turn and a bunching time of $<0.04 \mathrm{~s}$, bunches with rms length of $<0.6 \mathrm{~m} \quad(<2 \mathrm{~ns})$ can be obtained in a simple simulation. (see fig. 2) The rms energy width is then increased from $2 \mathrm{MeV}$ to $100 \mathrm{MeV}$ (full width $\pm 300 \mathrm{MeV}$ ). The faster 
bunching time indicates that other rf bunching scenarios (i. e., multi-harmonic, barrier bucket), may be implemented. After bunching, a $53 \mathrm{MHz}$ rf system could be turned on to hold the bunch length for the extraction period.

In the parameter lists we have not included a correction for the probability that a lower energy proton source is somewhat more efficient at lower energies $(\sim 10 \mathrm{GeV}$ rather than $56 \mathrm{GeV})$ in the production of low-energy pions. All other parameters equal, the $56 \mathrm{GeV}$ case should be $\sim 30 \%$ less efficient.

Thus another path to a muon collider would use the $8 \mathrm{GeV}$ Linac, directly fed into an $8 \mathrm{GeV}$ Accumulator and Buncher, where the pulse is charge-exchange injected and then bunched into a small number of short intense bunches. To a first order these machines could have the parameters of the Fermilab antiproton accumulator and debuncher.[3] At initial Project $X$ parameters the $8-\mathrm{GeV}$ intensity is limited to $0.36 \mathrm{MW}$. To obtain $2 \mathrm{MW}$ power the pulse length could be increased by a factor of 3 and the pulse rate increased from 5 to $10 \mathrm{~Hz}$. The accumulator/buncher system must then be able to bunch the beam at $\sim 0.1 \mathrm{~s}$ timescales.

\section{Potential Upgrade Paths}

The scenario could be upgraded by increasing the proton linac pulse length or peak current; the current scenario estimates that the Project-X linac power could be tripled. In that case the $56 \mathrm{GeV}$ power could also be tripled, obtaining $\sim 7 \mathrm{MW}$. This could be transformed into a luminosity increase in the Collider, potentially increasing luminosity by an order of magnitude. If all other parameters are kept the same, the beam-beam tune shift would increase to $\sim 0.16$, and the scenario may actually become beam-beam limited. 


\section{References}

[1] D. McGinnis (ed.), et al., "Accelerator Issues of Project X", Fermilab internal report, $8 / 7 / 2007$.

[2] R. B. Palmer, et al. "A Complete Scheme of Ionization Cooling for a Muon Collider", PAC07 Proc., Albuquerque NM, p. 3193 (2007).

[3] C. Ankenbrandt and M. Popovic, undocumented (2007).

Table 1: Collider Parameters.

\begin{tabular}{|l|l|l|l|}
\hline Parameter & Symbol & Baseline Value & \\
\hline Collision Energy & $2 \mathrm{E}_{\mu}$ & $4 \mathrm{TeV}$ & \\
\hline Luminosity & $\mathrm{L}$ & $4 \times 10^{34} \mathrm{~cm}^{-2}-\mathrm{s}^{-1}$ & \\
\hline Collider cycle frequency & $\mathrm{f}_{\mathrm{o}}$ & $15 \mathrm{~Hz}$ & \\
\hline$\mu$ 's per collider bunch & $\mathrm{N}_{\mu}$ & $1.2 \times 10^{12}$ & \\
\hline Transverse emittance & $\varepsilon_{\mathrm{N}}$ & $25 \quad \pi \mathrm{mm}-\mathrm{mr}$ & \\
\hline Collision parameter & $\beta^{*}$ & $0.003 \mathrm{~m}$ & \\
\hline Beam-Beam tune shift & $\delta \mathrm{v}$ & 0.052 & \\
\hline Beam size at collision & $\sigma_{\mu}$ & $2 \mu$ & \\
\hline Long. Emittance & $\varepsilon_{\mathrm{L}}$ & 70 & \\
\hline Momentum spread & $\delta \mathrm{p} / \mathrm{p}$ & $0.12 \%$ & \\
\hline Storage turns & $\mathrm{n}_{\mathrm{s}}$ & 900 & \\
\hline & & & \\
\hline Proton Driver parameters & & & \\
\hline Proton Energy & $\mathrm{E}_{\mathrm{p}}$ & $56 \mathrm{GeV}$ & \\
\hline Driver Frequency & $\mathrm{f}_{\mathrm{p}}$ & $1.67 \mathrm{~Hz}$ & \\
\hline Protons/driver cycle & $\mathrm{n}_{\mathrm{B}} \mathrm{N}_{\mathrm{p}}$ & $1.68 \times 10^{14}$ & \\
\hline Driver Power & $\mathrm{P}_{\mathrm{p}}$ & $2.5 \mathrm{MW}$ & \\
\hline Number of compressed bunches & $\mathrm{n}_{\mathrm{B}}$ & 9 & \\
\hline Intensity per bunch & $\mathrm{N}_{\mathrm{p}}$ & $1.87 \times 10^{13}$ & \\
\hline Muons per initial proton & $\mu / \mathrm{p}$ & 0.065 & \\
\hline & & & \\
\hline & & & \\
\hline
\end{tabular}




\section{Main Injector Cycle: 8 to $56 \mathrm{GeV}, 0.6 \mathrm{~s}$ Period}

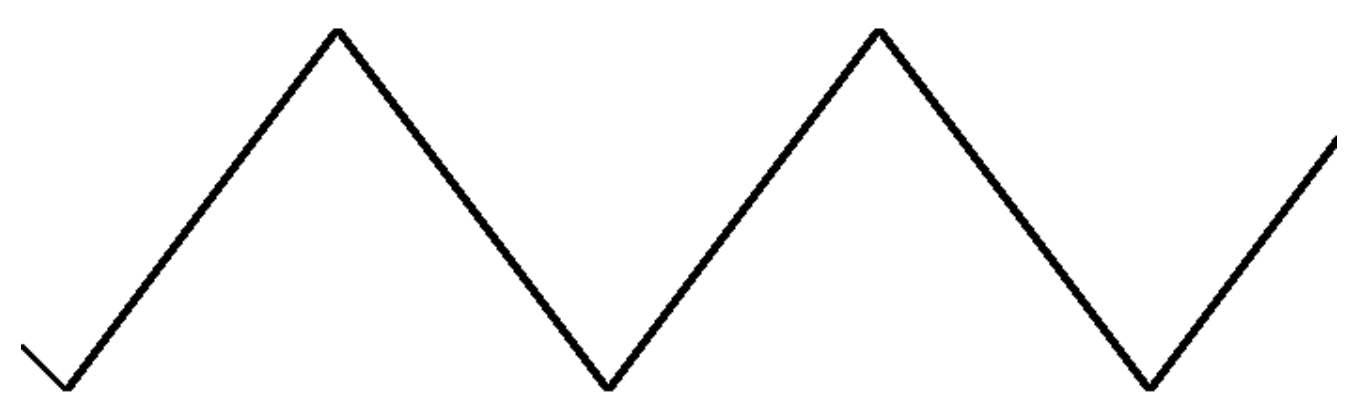

Recycler Intensity: 3 linac pulses

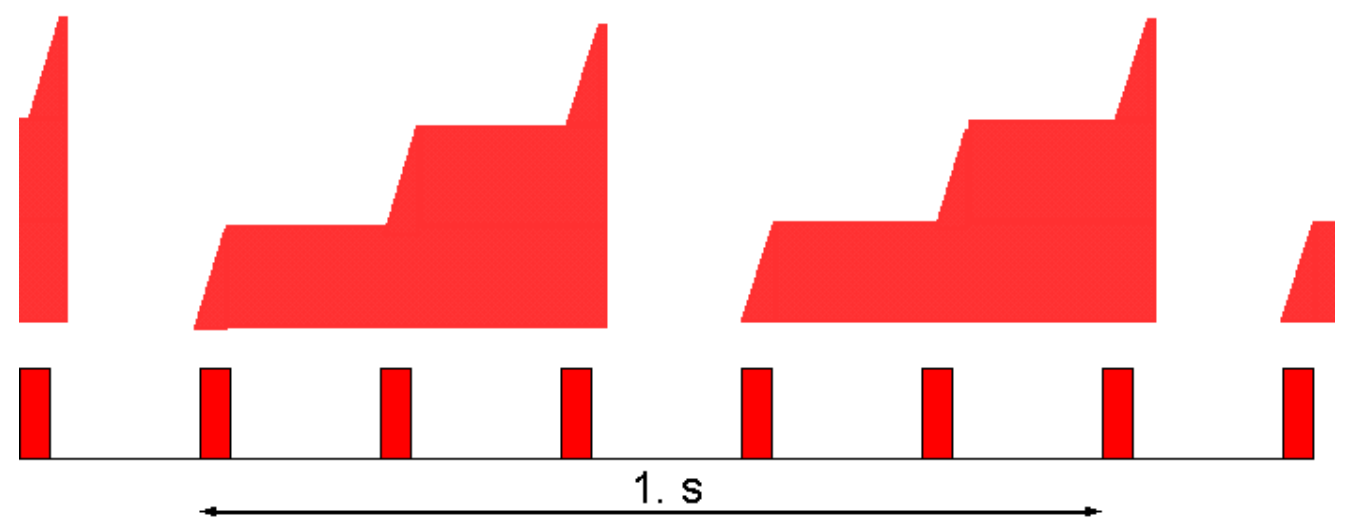

$8 \mathrm{GeV}$ Linac: $1 \mathrm{~ms}$ pulses at $5 \mathrm{~Hz}$

Fig. 1. Timeline obtained with an $8 \mathrm{GeV}$ linac, pulsing at $5 \mathrm{~Hz}$, filling the Recycler with three pulses. The resulting beam is transferred into the Main Injector with acceleration to $56 \mathrm{GeV}$, fast extraction into the Buncher, and reset occurring in 0.6s. 


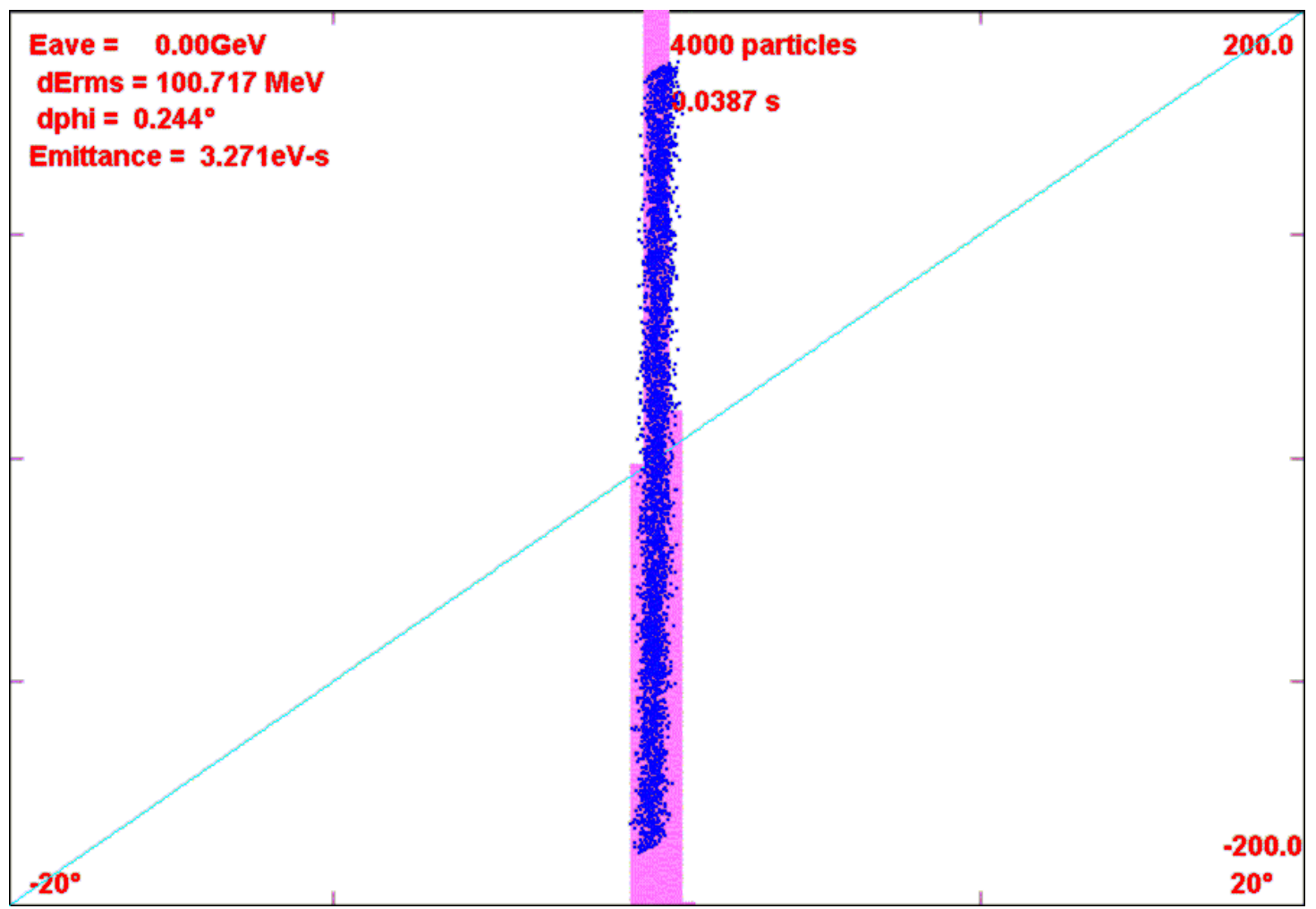

Fig. 1. Beam after bunching in a buncher ring with $1 / 4$ the circumference of the Main Injector. At parameters of $\gamma_{\mathrm{t}}=18$, a sawtooth $\mathrm{h}=9 \mathrm{rf}$ system of 0 to $0.4 \mathrm{MV} /$ turn and a bunching time of $<0.04 \mathrm{~s}$, bunches with rms length of $<0.6 \mathrm{~m}(<2 \mathrm{~ns})$ can be obtained in a simple simulation. 